\title{
In Situ TEM Observation of Atomic-level Shear Deformation for Nano-Tribological Research
}

\author{
T. Sato, ${ }^{*}$ T. Ishida, ${ }^{*}$ L. Jalabert, ${ }^{* *}$ and H. Fujita*
}

* The University of Tokyo, Institute of Industrial Science, 4-6-1 Komaba, Meguro-ku, Tokyo 1538505, Japan

** CNRS-IIS, The University of Tokyo, Institute of Industrial Science, 4-6-1 Komaba, Meguro-ku, Tokyo 153-8505, Japan

Monitoring the atomic-level shear deformation while detecting the shear force in real-time is an important step to elucidate the mechanism of lubrication. This is because the frictional interface is composed of multiple tiny shear deformations. However, observing the nano-scale shear deformation is a great challenge due to some experimental difficulties. In this research, we have developed a technique to combine MEMS (Micro Electro Mechanical Systems) technology with TEM (Transmission Electron Microscopy) ${ }^{(1,2)}$ to monitor shear deformation and shear force simultaneously.

Friction at the macroscopic level is attributed to the collective behavior of microscopic shear deformations. Therefore, a fundamental understanding of friction can be achieved by observing single contact points ${ }^{(3)}$. Some groups have combined TEM with AFM techniques to observe nanoscale deformation at a frictional interface ${ }^{(4)}$. Such TEM/AFM experimental systems are based on piezo actuation. The piezo actuator can produce nano-scale motion however, suffers from poor stability. Therefore, we used a MEMS electrostatic actuator instead of piezo actuators to achieve stable actuation.

In the experiments, using the MEMS electrostatic actuator, opposing tips were brought into contact. After forming a contact, one tip was actuated laterally slow enough to maintain the contact between the tips. Shear deformation of Ag junction was observed by TEM as shown in the extracted pictures from real time video. Fig.1(a) is the initial shape of Ag junction soon after the contact. The dark part in Fig. 1 corresponds to the silver tips and the light part corresponds to vacuum. The tip on the left side of the TEM image was the fixed edge and right side was actuated in the arrow direction in Fig.1(a). Shear deformation was observed as shown in Fig.1(b) (k). The junction was fractured from Fig.1(k) to Fig.1(1). As a result, we have succeeded in observing nano-scale deformation and fracture process in real time. Furthermore the shear force was simultaneously detected as shown in Fig.2.

To sum up, we have succeeded in observing atomic-level deformation and detecting the shear force at sub-nN order. We believe this work is a significant step to help revealing the mechanism of friction at nano-scale.

References

[1] Takaaki, S., et al, J. Phys.: Conf. Ser. 258, 2010, 012005

[2] Tadashi, I., et al, Journal of Micromechanics and Microengineering, 20, 2010, 075011

[3] C., M., Mate, Oxford university press, 2008, ISBN 978-0-19-852678-0

[4] Tokushi, K., et al, Nanotechnology 21, 2010, 495706 


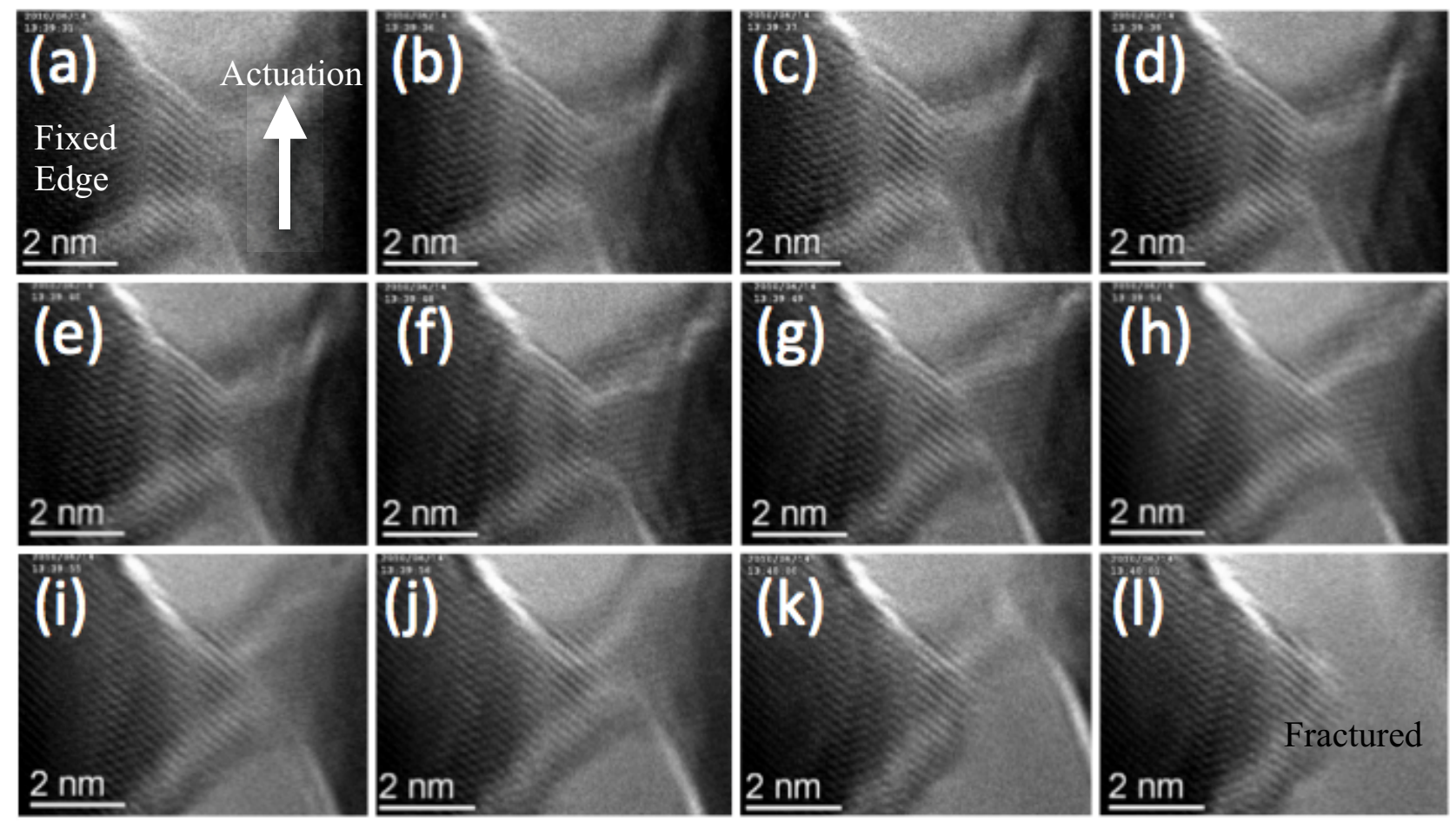

Fig.1 Ag shear deformation. TEM pictures were extracted from real time video. (a) is an initial shape of Ag junction. The junction deformed as shown in (b) (k). Fractured as shown in (l)

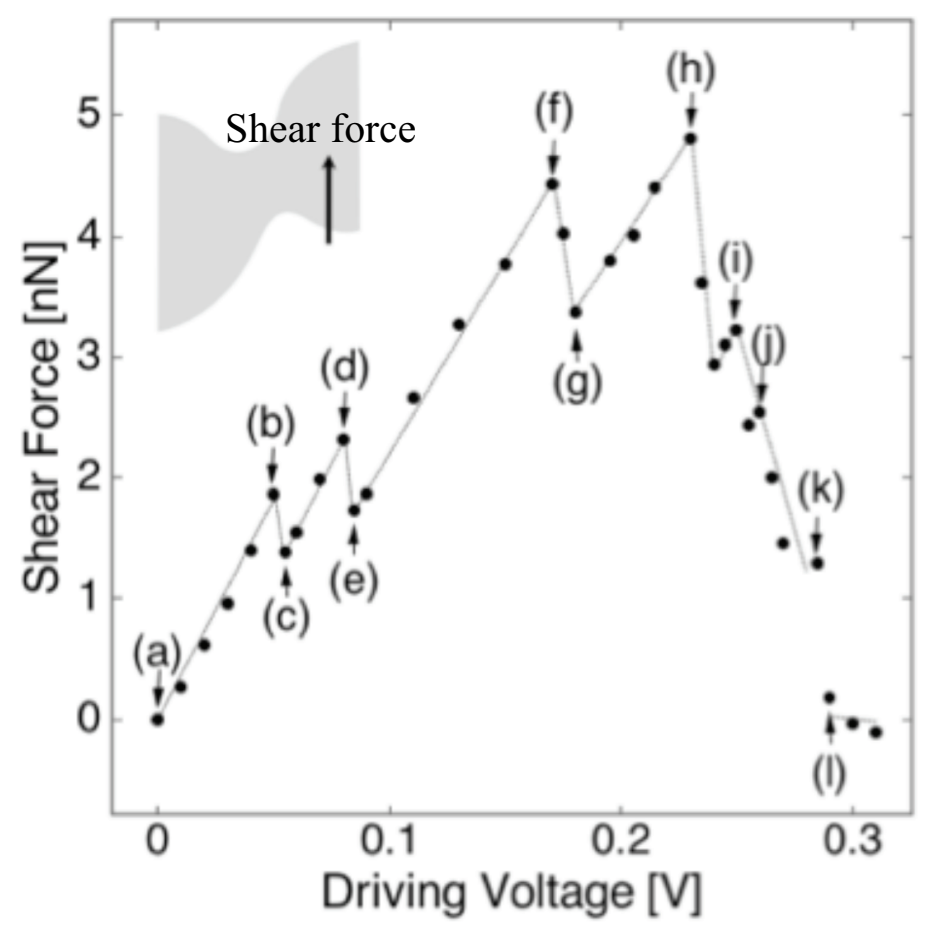

Fig.2 Displacement Shear displacement vs. shear force for shear testing of Ag nano junction. The arrows (a)-(1) correspond to the images of Fig.1. 